\title{
Prinzipien der Wortschreibung im Deutschen und Englischen am Beispiel der Schreibdiphthonge und der Doppelkonsonanten ${ }^{1}$
}

\begin{abstract}
In diesem Aufsatz geht es um einen Vergleich der Prinzipien der Wortschreibung im Englischen und Deutschen. Konkret werden Schreibdiphthonge und Doppelkonsonanten behandelt. Beide Phänomene eignen sich gut, um Prinzipien zu verstehen, nach denen die Wortschreibung funktioniert: So lassen sich Schreibdiphthonge nicht immer so aussprechen, wie es die einzelnen Vokalbuchstaben suggerieren, das heißt, sie sind nicht immer über die entsprechenden Graphem-Phonem-Korrespondenzen der einzelnen Segmente zu interpretieren, etwa <ei> für / ai/ im Deutschen und $<$ ea $>$ für /i / im Englischen. Auf einer, höheren'Ebene (der silbischen) zeigen sich aber systematische Züge, die in beiden Sprachen vergleichbar sind. Auch die Schreibungen der Doppelkonsonanten sind nicht einfach auf der Segmentebene zu verstehen, sondern sie ergeben sich aus einem Zusammenspiel der silbischen, der suprasegmentalen und der morphologischen Ebene. In beiden Sprachen wirken Prinzipien auf allen diesen Ebenen, aber zum Teil auf unterschiedliche Art und Weise.
\end{abstract}

\section{Die Prinzipien der Wortschreibung}

Sowohl für das Deutsche als auch für das Englische sind zumindest folgende Schreibprinzipien anzunehmen: segmentale, silbische, suprasegmentale, morphologische, graphotaktische und syntaktische. Die genannten Prinzipien sind dabei als Gruppen zu verstehen, so gibt es beispielsweise mehrere morphologische, mehrere silbische Prinzipien usw. Welche Prinzipien genau anzunehmen sind, ist dabei eine schwierige Frage. Allzu oft werden ad hoc Prinzipien für Einzelfälle formuliert. Um dies zu verhindern, geht es auch um die Wirkungsweise von Prinzipien. Typischerweise kann die Wirkung in der Schrift sichtbar sein. Aus rein methodischen Gründen ist zwischen einer, expliziten' und einer, impliziten ' Wirkung zu unterscheiden. Dies erläutern wir an einem morphologischen Prinzip, nämlich der Stammkonstanzschreibung: Die Schreibung des Substantivs Mann mit <nn> zeigt die Beziehung zu $<$ Männer $>$; ohne diese Beziehung wäre eine Schreibung $<$ man $>$ angemessen, die es für das Pronomen ja gibt. <nn $>$ ist in $<$ Mann $>$ also ausschließlich über die Stammkonstanzschreibung zu begründen. Das

Es handelt sich um erste Ergebnisse eines von der DFG geförderten Forschungsprojektes „Prinzipien der Wortschreibung im Deutschen und Englischen'. 
wäre eine, explizite ' Wirkung eines morphologischen Prinzips und ist typisch für Doppelkonsonantenschreibungen bei Einsilbern im Deutschen. Bei einem Paar wie Tisch - Tische hingegen findet sich ebenso Stammkonstanzschreibung; sie ist aber implizit, denn sowohl tisch als auch tische ergeben sich bereits ausschließlich aus Phonem-Graphem-Korrespondenzen. Das Stammkonstanzprinzip greift nicht sichtbar ein, es verändert nicht die Schreibung. In Abschnitt 1.5 werden diese Beispiele noch einmal aufgegriffen.

Grundsätzlich sind im Deutschen und Englischen die Schreibprinzipien vergleichbar; Unterschiede finden sich in den konkreten Auswirkungen. Mit den oben genannten Prinzipien lässt sich die Schreibung von über 95\% des nativen deutschen Wortschatzes erfassen (siehe Eisenberg 2006, S. 301350). Ähnliche Schätzungen über das Englische fehlen noch; auch ist die Wirkungsweise der Schreibprinzipien noch nicht in der gleichen systematischen Weise wie für das Deutsche erfasst.

Wir werden im Folgenden die genannten Prinzipien der Wortschreibung kurz für das Deutsche und das Englische erläutern. Anschließend werden wir genauer die Schreibdiphthonge und die Doppelkonsonanten untersuchen; zuerst für das Deutsche und dann für das Englische. Ein Punkt wird gesondert behandelt, und zwar, verbotene Wortenden'; ein Phänomen, auf das man immer wieder stößt, wenn man sich mit der Schreibung des Englischen befasst.

\subsection{Segmentale Prinzipien}

Mit diesen Prinzipien sind weitgefasst die Laut-Buchstaben-Zuordnungen gemeint, und zwar auf der segmentalen Ebene. Wir möchten die Diskussion kurz für unsere Zwecke zielgerichtet zusammenfassen. Die grundlegende Idee ist, dass man zum einen Phonem-Graphem-Korrespondenzen definieren kann für die so genannte Schreib- oder Kodierungsrichtung, und zum anderen Graphem-Phonem-Korrespondenzen für die Lese- oder Rekodierungsrichtung (Neef 2005). Es ist interessant zu fragen, in welche Richtung mehr Eindeutigkeit erreicht werden kann, in welchen Richtungen Mehrdeutigkeiten vorherrschen und ob diese systematisch zu beschreiben sind oder nicht. So ist zum Beispiel die Schreibung von Schwa im Deutschen als $\langle\mathrm{e}\rangle$ eindeutig; die Lesung von $\langle\mathrm{e}\rangle$ auf der segmentalen Ebene aber nicht, denn es kann als Schwa, als / $\varepsilon$ / oder als /e/gelesen werden bzw. ein Wort wie beten wird typischerweise ohne Schwa realisiert, $<\mathrm{e}>$ sichert hier eine graphematische Silbigkeit. Auf der anderen Seite kann /n/ offenbar sowohl als $<\mathrm{n}>$ als auch als $<\mathrm{nn}>$ geschrieben werden; beides wird nicht auf der segmentalen Ebene, sondern entweder auf der silbischen, der suprasegmentalen oder auf der morphologischen Ebene erfasst; sowohl $<\mathrm{n}>$ als auch $<$ nn $>$ können aber als /n/ gelesen werden. 
Für das Deutsche kann man mit Eisenberg (2006, S. 306) zum Beispiel 20 Konsonantengrapheme und 9 Vokalgrapheme annehmen, also 29 Grapheme. Als Phoneme findet Eisenberg 21 konsonantische und 16 vokalische (2006, S. 90-98). Becker (1996) nimmt keinen Gespanntheitsunterschied der Vokale an, sondern den Silbenschnitt. Damit ergibt sich eine Anzahl von 9 vokalischen Phonemen, 8 Vollvokale und Schwa. Die Anzahl von Graphemen und Phonemen ähnelt sich durchaus. Das Schriftsystem des Deutschen ist dennoch auf der segmentalen Ebene unterspezifiziert, so bezieht sich das Graphem $<\mathrm{b}>$ primär auf den Laut [b] und sekundär auf [p] (Neef 2005, S. 227), das Graphem <a> sowohl auf [a] als auch auf [a] (ebd.).

\begin{tabular}{|c|c|c|c|}
\hline \multicolumn{2}{|l|}{ Vokale $(16+3)$} & \multicolumn{2}{|l|}{ Konsonanten $(21+2)$} \\
\hline Monophthonge & Diphthonge & einfach & Affrikate $^{2}$ \\
\hline 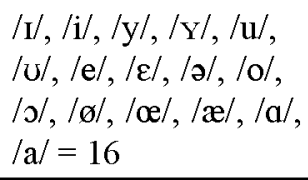 & $\begin{array}{l}/ \mathrm{ai} /, / \mathrm{oi} /, / \mathrm{au} / \\
=3\end{array}$ & $\begin{array}{l}/ \mathrm{p} /, / \mathrm{b} /, / \mathrm{t} /, / \mathrm{d} /, / \mathrm{k} /, / \mathrm{g} / \\
/ \mathrm{p} /, / \mathrm{f} /, / \mathrm{v} /, / \mathrm{s} /, / \mathrm{s} /, / \mathrm{z} / \\
/ \mathrm{z} /, / \mathrm{c} /, / \mathrm{d} /, / \mathrm{h} /, / \mathrm{m} /, / \mathrm{n} /, \\
/ \mathrm{y} /, / 1 /, / \mathrm{R} /=21\end{array}$ & $\begin{array}{l}/ \mathrm{ts} /, / \mathrm{pf} / \\
=2\end{array}$ \\
\hline
\end{tabular}

Abb. 1: Phoneme im Deutschen (nach Eisenberg 2006, S. 90-98)

\begin{tabular}{|c|c|c|c|}
\hline & einfach & komplex & Pseudogeminaten \\
\hline $\begin{array}{l}\text { Konsonanten } \\
(20+2)\end{array}$ & 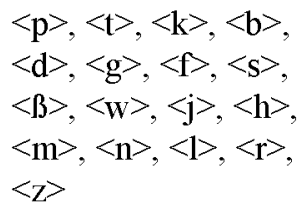 & $\begin{array}{l}<\mathrm{ch}>,<\mathrm{sch}> \\
<\mathrm{qu}>\end{array}$ & $\begin{array}{l}\langle\mathrm{tz}\rangle,\langle\mathrm{ck}\rangle,{ }^{3} \\
\text { siehe Abschnitt } 3\end{array}$ \\
\hline Vokale $(8+6)$ & $\begin{array}{l}<\mathrm{a}\rangle,\langle\mathrm{e}\rangle,\langle\mathrm{i}\rangle,\langle\mathrm{o}\rangle, \\
<\mathrm{u}\rangle,\langle\ddot{\mathrm{a}}\rangle,\langle\ddot{\mathrm{o}}\rangle,\langle\ddot{\mathrm{u}}\rangle\end{array}$ & $\begin{array}{l}<\mathrm{ei}>,<\mathrm{ai}>,<\mathrm{eu}>\text {, } \\
<\mathrm{au}>,<\mathrm{ie}> \\
\text { (<äu> morph., } \\
\text { siehe Abschnitt } \\
2.1)\end{array}$ & \\
\hline
\end{tabular}

Abb. 2: Grapheme im Deutschen (nach Eisenberg 2006, S. 306, 312)

Im Englischen sind die Zahlenverhältnisse bei den Konsonanten vergleichbar, so nimmt Giegerich 24 Konsonantenphoneme an (1992, S. 34). Demgegenüber stehen 27 angenommene Konsonantengrapheme (Venezky spricht hier von ,relational units“, siehe 1999, S. 82). Bei den Vokalen ist die Bestimmung des Phonem-Graphem-Bestandes schon schwieriger: Hier finden

\footnotetext{
Der Phonemstatus der Affrikaten ist umstritten. Eisenberg nimmt sie so nicht an; sie werden hier angegeben, weil sie auch im Englischen angegeben sind und weil ihre Schreibung sehr interessant ist.

3 Dies sind Graphemketten, deren einzelne Auflistung aber lohnt.
} 
sich sechs einfache Vokalgrapheme (die fünf Vokalbuchstaben und $\langle y\rangle$ ) und eine ganze Reihe von komplexen. Dabei fallen unter die komplexen sowohl ,Schreibdiphthonge' wie $<\mathrm{ea}>($ seat $),<\mathrm{ou}>($ soup $)$, , diskontinuierliche $^{6}$ Diphthonge wie $<$ i_e $>(l i k e)^{4}$ als auch Doppelvokalschreibungen wie $<\mathrm{ee}>$. Phonologisch sind nach Ladefoged (2006, S. 39) 11 einfache Vokalphoneme für das AE bzw. 12 für das BE (ebenso Giegerich 1992, S. 45) und fünf (bzw. Giegerich drei) Diphthonge ${ }^{6}$ anzunehmen. Die primären Beziehungen sind auf jeden Fall im Englischen schwieriger, wie auch schon die Korpusanalysen zu den Phonem-Graphem-Korrespondenzen von Hanna et al. (1966) sowie die Graphem-Phonem-Korrespondenzen von Dewey (1970) und auch die Fallstudien von Kessler/Treiman (2001) belegen. Zu den Diphthongen siehe auch Abschnitt 2.2.

\begin{tabular}{|c|c|c|c|}
\hline \multicolumn{2}{|l|}{ Vokale $(11+5)$} & \multicolumn{2}{|l|}{ Konsonanten $(22+2)$} \\
\hline Monophthonge & Diphthonge & einfach & Affrikate \\
\hline 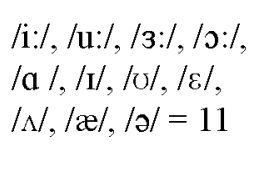 & $\begin{array}{l}/ \mathrm{e} /, / \mathrm{ov} /, / \mathrm{oI} /, \\
/ \mathrm{a} / /, / \mathrm{au} /=5\end{array}$ & $\begin{array}{l}\mathrm{h} /, / \mathrm{d} /, / \mathrm{f} /, / \mathrm{g} /, / \mathrm{h} /, / \mathrm{j} /, / \mathrm{k} /, \\
/ \mathrm{l} /, / \mathrm{m} /, / \mathrm{n} /, / \mathrm{y} /, / \mathrm{p} /, / \mathrm{r} /, / \mathrm{s} /, \\
/ \mathrm{J} /, / \mathrm{t} /, / \mathrm{v} /, / \mathrm{w} /, / \mathrm{z} /, / 3 /, / \theta /, \\
/ \circlearrowright /=22\end{array}$ & $/ \mathrm{tg} /, / \mathrm{d}_{3} /=2$ \\
\hline
\end{tabular}

Abb. 3: Mögliche Phoneme im Englischen

\begin{tabular}{|c|c|c|c|}
\hline & einfach & komplex & Pseudogeminaten \\
\hline $\begin{array}{l}\text { Konsonanten } \\
(27+2)\end{array}$ & $\begin{array}{l}<\mathrm{b}, \mathrm{c}, \mathrm{d}, \mathrm{f}, \mathrm{g}, \mathrm{h}, \mathrm{j}, \mathrm{k} \\
\mathrm{l}, \mathrm{m}, \mathrm{n}, \mathrm{p}, \mathrm{r}, \mathrm{s}, \mathrm{t}, \mathrm{v}, \mathrm{w} \\
\mathrm{x}, \mathrm{y}, \mathrm{z}>=20\end{array}$ & $\begin{array}{l}<\mathrm{ch}, \mathrm{gh}, \mathrm{gu}, \mathrm{cqu}^{7} / \\
\mathrm{qu}, \mathrm{sh}, \mathrm{th}, \text { wh }>=7 \\
\text { (ohne Allographen) }\end{array}$ & $<\operatorname{tch}\rangle,\langle\operatorname{dg}\rangle$ \\
\hline \multirow[t]{2}{*}{ Vokale (25) } & \multirow[t]{2}{*}{$<\mathrm{a}, \mathrm{e}, \mathrm{i}, \mathrm{o}, \mathrm{u}, \mathrm{y}>=6$} & $\begin{array}{l}<\text { ai/ay, au/aw, ea, } \\
\text { ee, ei/ey, eo, eu/ew, } \\
\text { ie, oa, oi/oy, oo, } \\
\text { ou/ow, ui, uy> }>14 \\
\text { (ohne Allographen) }\end{array}$ & \\
\hline & & $\begin{array}{l}<\text { a_e, e_e, i_e, o_e, } \\
\text { u_e }>=5\end{array}$ & \\
\hline
\end{tabular}

Abb. 4: Mögliche Grapheme im Englischen

4 Diese sind mit der Fußstruktur von Primus (2011) nicht als eigene Grapheme anzusehen, siehe auch Abschnitt 2.

$5 \mathrm{Im} \mathrm{BE}$ kommt $/ \mathrm{D} /$ in pot hinzu.

6 Dem Diphthong /ju/ in mate kommt aufgrund des Glides eine Sonderstellung zu. Die drei zusätzlichen Diphthonge im BE /Ia/, /عa/, und /aə/ sind Resultat einer Neutralisierung zu Schwa vor/r/ (Beispiel: hairwird im BE/hea/gelesen, im AE hingegen/her/) und bleiben hier auBer Betracht.

Zum Beispiel in acquaint. 
Bei den hier angenommenen Auflistungen ergeben sich also 52 Grapheme und 40 Phoneme, das Verhältnis bei den Konsonanten ist dabei 22 Konsonantenphoneme zu 27 Konsonantengraphemen. Bei den Vokalen hingegen 16 Phoneme zu 25 Graphemen. Bei einer Zählung, wie sie nach Eisenberg vorgenommen werden kann, ist im Deutschen die Zahl der Vokalphoneme höher als die der Grapheme, mit der Annahme des Silbenschnitts ist sie annähernd gleich (8 o. 9 Phoneme, 8 o. 9 Grapheme). Die vorliegende Auflistung für das Englische zeigt zunächst ein umgekehrtes Verhältnis. Die hohe Zahl der Vokalgrapheme ergibt sich aus den häufigen Schreibdiphthongen, weswegen wir diese in Abschnitt 2.2 genauer untersuchen. Ohne allzu sehr vorzugreifen, ist Folgendes offensichtlich: Im nativen Wortschatz des Deutschen korrespondiert ein Sprechdiphthong immer mit einem Schreibdiphthong und ein Schreibdiphthong korrespondiert meistens mit einem Sprechdiphthong (Ausnahme $<\mathrm{ie}>$ ). Im Englischen gilt dies weder für die eine noch für die andere Richtung: Der Schreibmonophthong $<i>$ korrespondiert mit dem Sprechdiphthong / ar / und der Sprechdiphthong / i / mit dem Schreibdiphthong $\langle e a\rangle$. Die hier vorgenommene Auflistung für das Englische ist umfangreich. Die Hinzuziehung weiterer Schreibprinzipien, insbesondere der silbischen und suprasegmentalen, könnte zu einer Reduzierung des Grapheminventars führen, insbesondere bei den komplexen Vokalgraphemen ist damit zu rechnen, wenn Prinzipien auf höheren Ebenen hinzugezogen werden.

\subsection{Silbische und suprasegmentale Schreibprinzipien}

Eisenberg (2006, S. 310-319) fasst unter die silbischen Schreibungen Prinzipien zusammen, die speziell den Anfangsrand, den Kern und den Endrand betreffen. Mit der Einführung des graphematischen Fußes durch Evertz (2011) und Primus (2011) kann hier aber zwischen silbischen und suprasegmentalen Schreibungen unterschieden werden. Silbische Schreibungen beziehen sich dann auf die Silben selbst, suprasegmentale auf das Gewicht der Silben. Vor allem die Besonderheiten bei der Interpretation des graphematischen Silbenkerns (der Bezug auf gespannte und ungespannte Vokale) sind dann eher suprasegmentale Schreibungen; damit wird der Silbenschnitt - wie ihn Becker (1996) explizit für die Phonologie annimmt zu der unterscheidenden Einheit in Minimalpaaren wie / mits/ und / mits/. Ein silbisches Schreibprinzip ist zum Beispiel die Einhaltung des Graphematischen Silbenbaugesetzes (für das Deutsche Fuhrhop/Buchmann 2009, für das Englische Fuhrhop/Buchmann/Berg im Erscheinen); Prinzipien, die die Schreibsilbe hervorheben (wie zum Beispiel Roemhelds 1955, S. 73 77) Beobachtung, dass das Auftreten vom Dehnungs- $b$ auch abhängig von den Anfangsrändern ist, so zum Beispiel Schule und nicht *Schuble. Auch die interne Organisation der Schreibdiphthonge ist ein silbisches Prinzip, dazu mehr in Abschnitt 2. 
Primus (2011) und Evertz (2011) führen den graphematischen Fuß ein, und zwar ebenfalls an den Beispielsprachen Englisch und Deutsch.

Zunächst zum phonologischen Fuß: Sowohl das Deutsche als auch das Englische haben Silben mit unterschiedlichem Gewicht (Restle/Vennemann 2001, S. 1328). Schwere Silben haben typischerweise einen verzweigenden Kern, leichte Silben haben diesen nicht. Ein verzweigender Kern meint zwei Positionen im Kern, diese können besetzt sein durch einen Kurzvokal und einen Konsonanten oder durch einen Diphthong oder durch einen Langvokal, der dann binär ist, also zwei Positionen besetzt. Entsprechend hat weder das Deutsche noch das Englische „,betonte Kurzvokale in offener Silbe" (Restle/Vennemann 2001, S. 139). Jeder Fuß enthält eine schwere Silbe und eine oder mehrere unbetonte Silben (Primus 2010, S. 23).

Dieses Konzept übertragen Evertz (2011) und Primus (2010, 2011) auf die Graphematik. Auch die Graphematik hat dann verzweigende Kerne. Zwei Positionen ergeben sich entweder durch einen (Schreib-)Diphthong (Scbrei-ber) oder durch einen Vokal- und einen Konsonantenbuchstaben (Filler, Fil-ter). Bei Primus (2011, S. 4) ist ein Vokalbuchstabe, der in einer offenen Vollsilbe steht, binär. Segmental ist dies nicht zu erkennen, aber offene Silben sind zu erkennen und so ergibt sich die Binarität für die Übertragung in die phonologische Fußstruktur, unabhängig davon, ob dies graphematisch als binär beschrieben wird. Es ist also die Rekonstruktion des Silbenschnitts.

In der Neuerung unterscheiden Primus (2011) und Evertz (2011) konsequent den kanonischen Fuß von den nicht-kanonischen Füßen. Der kanonische graphematische Fuß ist sowohl für das Deutsche als auch für das Englische der trochäische mit einer speziellen zweiten Silbe: Phonologisch ist die zweite Silbe unbetonbar, sie enthält ein Schwa oder einen silbischen Konsonanten, graphematisch ist sie leicht und enthält typischerweise ein $<\mathrm{e}>$. Alle anderen Füße sind dann nicht-kanonisch.

Die zweisilbige Struktur ist im englischen Schriftsystem viel stärker vertreten als im englischen Lautsystem, Schreibungen wie like, gate, sale usw. sind im Englischen graphematisch zweisilbig und phonologisch einsilbig; graphematisch sind dies kanonische Füße. Durch die Öffnung der graphematischen Silbe kann der Leser erkennen, dass der Vokal der ersten Silbe phonologisch zwei Positionen besetzt, also entweder ein Langvokal oder ein Diphthong ist (Primus 2011, S. 3).

Im Deutschen hingegen kann man weitgehend davon ausgehen, dass die phonologische Silbenzahl und die graphematische Silbenzahl in den meisten Fällen übereinstimmen (Tische, Wiese, Männer). Ausnahmen sind Schreibungen wie Dirndl (graphematisch nur ein Silbenkern). Interessant ist hier das silbeninitiale $<\mathrm{h}>$ wie in drehen, Ruhe usw. Die graphematische Zweisilbigkeit wird gesichert, auch wenn in einzelnen Formen lautlich die Einsilbigkeit realistischer erscheint, wie in drehen. ${ }^{8}$

Innerhalb des Flexionsparadigmas sind aber spätestens mit den Konjunktivformen (drehe, drehest usw.) auch entsprechende zweisilbige Formen zu finden. 
1.3 Morphologische Schreibungen

Die expliziten Wirkungen der Stammkonstanz im Deutschen zeigen sich bei der Übernahme von Dehnungs- $b$ und silbeninitialem $\mathrm{h}$ (dehnt, dreht), der Nichtrealisierung der Auslautverhärtung in der Schrift (Hund statt ${ }^{*} H$ unt) und der Übernahme der Silbengelenkschreibung in Nichtsilbengelenkpositionen (kïssen - kiusst). Im Englischen hingegen werden häufig Beispiele wie sign vs. signature, signal, muscle - muscular, autumn - autumnal, doubtindubitable (Meisenburg 1996, S. 22-30) angegeben; ,stumme Konsonantenbuchstaben (nicht segmental begründet) zeigen morphologische Verwandtschaft. Gerade bei Vokalschreibungen hält das Englische die Stammkonstanz nicht immer ein wie in pronounce - pronunciation, declaim - declamation, sheep shepherd, deep - depth (Venezky 2004, S. 146). Hier sind systematischere Untersuchungen nötig (so auch ebd., S. 148).

Systematisch scheint es im Englischen bei regelmäßigen Verben eine Suffixkonstanzschreibung bei -ed zu geben: Unabhängig von der phonologischen Silbigkeit ist es graphematisch immer silbisch (begged, jumped, washed). ${ }^{9}$ Venezky nimmt außerdem eine Konstanzschreibung für das Suffix $-s$ an (boys statt *boyz, wie es phonologisch nahegelegen hätte); die Silbigkeit unterscheidet er hier allerdings nicht (glass-es vs. boy-s). Hier verhält sich Englisch eher wie Deutsch: Die graphematische Silbigkeit bildet die phonologische Silbigkeit ab (Haus-es, Mann-es neben Auto-s, Mann-s). Aber auch das Deutsche zeigt eine Suffixkonstanzschreibung, wie in Männer zu sehen: Es wird mit -er (und nicht etwa mit - $a$ am Ende) geschrieben, weil es sich um ein Pluralsuffix handelt, das eben -er heißt.

Die expliziten Wirkungen der Morphemkonstanz (zusammenfassend für Stamm- und Affixkonstanz) sind in beiden Sprachen unterschiedlich; auf der Phänomenebene vergleichbar sind die Doppelkonsonanten, denn sie treten in beiden Sprachen auf; Unterschiede sind aber augenscheinlich, da im englischen Einsilber typischerweise keine Doppelkonsonanten auftreten (rennen - rennt vs. running - to run); diese werden im Abschnitt 3 behandelt. Hier wird sich zeigen, dass es auch ein morphologisches Schreibprinzip zur Komplexitätsanzeige gibt.

\subsection{Graphotaktische Schreibungen im Deutschen und Englischen}

Unter diesem Begriff sind Prinzipien zusammengefasst, die ausschließlich die Schriftseite betreffen. Venezky (2004, S. 194 f.) hat einige von ihnen als ,scribal constraints ${ }^{c}$ beschrieben, zum Beispiel die Tatsache, dass bestimmte Wortenden wie $\left\langle_{\mathrm{v}}\right\rangle$ im Englischen vermieden werden (siehe auch, Verbotene Wortenden', Abschnitt 4). Für das Deutsche einschlägig sind hier Prin-

Einige Schreibungen wie learnt, burnt zeigen diese Konstanz allerdings nicht, so auch Venezky (2004); für learnt findet sich inzwischen auch die Schreibung learned. 
zipien wie Roemhelds Ausführungen über das vermeintliche Dehnungs- $h$, das für die ideale Wortbreite von vier Buchstaben bei Autosemantika sorgt, zum Beispiel Mehl vs. schal (1955, S. 73-77) und Jespersens Drei-Buchstaben-Regel fürs Englische (siehe Abschnitt 3.2.3). Auch die Tatsache, dass komplexe Grapheme wie <sch $>$ im Deutschen oder $<$ sh $>$ im Englischen in Silbengelenkposition nicht verdoppelt werden, ist ein graphotaktisches Prinzip (*Tischsche, *fishshing).

1.5 Die angenommenen Prinzipien der Wortschreibung im Deutschen und Englischen
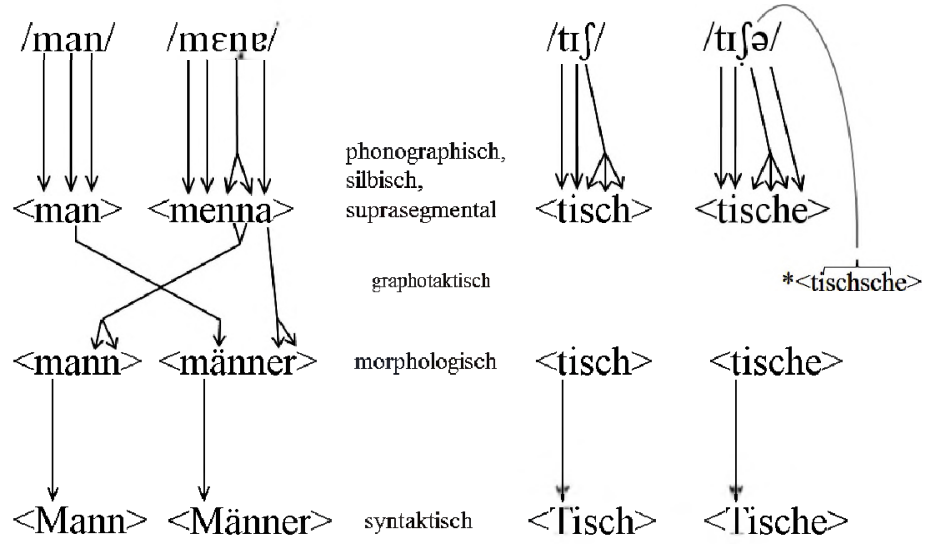

Abb. 5: Die Wortschreibung des Deutschen

Diese Beispiele zeigen die grundsätzlichen Prinzipien der Wortschreibung des Deutschen. Dabei sind rein abbildungstechnisch die segmentalen, silbischen und suprasegmentalen Prinzipien in einer Stufe erfasst; alle diese Prinzipien beinhalten einen Rückgriff auf die Lautstruktur. Die morphologischen Prinzipien betreffen sowohl die Stammschreibung (Doppel- $n$ in Mann) als auch die Suffixschreibung (-er in Männer). Die Verhinderung von * ischsche ist graphotaktisch, komplexe Grapheme werden nicht verdoppelt. In der Gegenüberstellung von Mann - Männer und Tisch - Tische sind, wie oben beschrieben, explizite und implizite Wirkungen zu erkennen, insbesondere bei den morphologischen Prinzipien. Die syntaktischen Prinzipien betreffen insbesondere die Groß- und Kleinschreibung und die Getrennt- und Zusammenschreibung; beide Phänomene werden im vorliegenden Aufsatz nicht weiter behandelt. Übertragen wir die Prinzipien aufs Englische an einigen Beispielen, und zwar an swimmer wegen der Doppelkonsonantenschreibung, late - later nach Primus (2011, S. 6) wegen des suprasegmentalen Prinzips und boy - boys nach Venezky (2004, S. 147) wegen der Suffixkonstanz. 


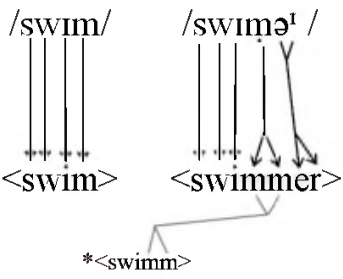

$<$ swim $>\quad<$ swimmer $>$

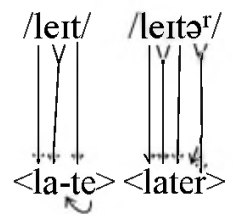

$<$ late $><$ later $>$

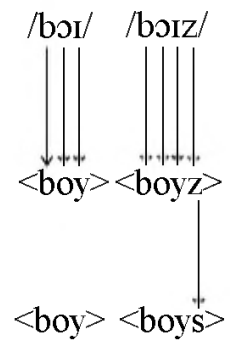

Abb. 6: Die Wortschreibung des Englischen

Offenbar wirkt das Stammkonstanzprinzip im Englischen bei swimmer - smim nicht; siehe dazu Abschnitt 3. In dem Beispiel late zeigt sich die Wirkung eines suprasegmentalen Prinzips: Es handelt sich bei /eI $-<\mathrm{a}>$ um eine mögliche segmentale Schreibung, und zwar fïr offene Silben (Venezky unterscheidet in diesem Zusammenhang, free ${ }^{6}$ und ,checked', siehe Abbildung 12). Die Silbe wird mit Hilfe von $<\mathrm{e}>$ graphematisch geöffnet, also $<$ la-te $>$, im Englischen hat $\langle\mathrm{e}\rangle$ am Wortende typischerweise diese suprasegmentale Wirkung und keine segmentale Lesart (außer in Eigennamen wie Nike). Bei boys ist zu erkennen, dass im Englischen ein morphologisches Prinzip bei der Suffixschreibung wirkt; ein Suffix wird möglichst gleich geschrieben; bzgl. der Stimmhaftigkeit ist $<s>$ an dieser Stelle unterspezifiziert. Die Prinzipien sind hiermit zusammenfassend beschrieben und wir kommen zu den konkreten Untersuchungen: den Schreibdiphthongen (und Doppelvokalschreibungen) und den Schreibungen der Doppelkonsonanten.

\section{Schreibdiphthonge und Doppelvokalschreibungen im Deutschen und Englischen}

Im Folgenden wird untersucht, wie sich Schreibdiphthonge innergraphematisch organisieren. Die Doppelvokalschreibungen $(\langle\mathrm{aa}\rangle,\langle\mathrm{ee}\rangle,\langle\mathrm{oo}\rangle)$ sind hier mit aufgenommen, da es interessante Zusammenhänge zu den Schreibdiphthongen gibt. Anschließend werden sie auf die Lautung bezogen.

\subsection{Schreibdiphthonge und Doppelvokalschreibungen im Deutschen}

Im Deutschen sind die komplexen graphematischen Silbenkernverbindungen recht gut beschrieben: Neben den Schreibdiphthongen <ai, ei, au, eu, ie, äu> kommen die Doppelvokalgrapheme <aa, ee, oo> vor. Die Doppelvokalgrapheme haben die gleichen Korrespondenzen wie die einzelnen für die gespannten Varianten. $<$ aa $>$ und $<$ oo $>$ sind in der Schreibung des Deutschen aber selten, <ee $>$ häufiger. $<$ äu $>$ ist eine morphologische Variante von $\langle$ eu $>$, also steht $|$ ä $\mid$ für $|\mathrm{e}|$ aus morphologischen Gründen, wie auch 
bei dem Monophthong (Äpfelwg. Apfelwie Häuser wg. Haus), unabhängig von dem folgenden $|\mathrm{u}| .<$ ie $>$ ist die Schreibung für /i/ und hier taucht es häufig schon in den Phonem-Graphem-Korrespondenzen auf bzw. bei Primus (2010) als suprasegmentale Schreibung. Bei den anderen vier Schreibdiphthongen ( $\langle\mathrm{ai}\rangle,\langle\mathrm{au}\rangle,\langle\mathrm{ei}\rangle,\langle\mathrm{eu}\rangle$ ) unterscheidet Eisenberg (2006, S. 312) nach typischen ersten $(|\mathrm{a}|,|\mathrm{e}|)$ und typischen zweiten (|i|,|u|) Bestandteilen; es ergibt sich ein vollständiges System.

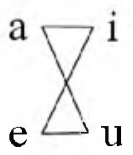

Abb. 7: System der Schreibdiphthonge im Deutschen

Im Deutschen sind Regelmäßigkeiten bei der Rekodierung aufgrund des Erstbestandteils anzugeben: Beim Erstbestandteil $|a|$ wird segmental rekodiert, beim Erstbestandteil $|\mathrm{e}|$ gerade nicht (siehe auch Berg/Fuhrhop im Erscheinen, S. 464). Allerdings ist in der einzigen ,Doppelvariante ${ }^{c}<a i / e i>$ (für /ai/) <ei> die häufigere Schreibung. Die nicht-segmentale Rekodierung vom Erstbestandteil $|\mathrm{e}|$ ist ein weiteres Indiz dafür, dass $|\mathrm{e}|$ in den Silbenkern drängt (so auch als Verschriftung von Schwa und bei <ie>, siehe Fuhrhop/Buchmann 2009, S. 148).

\subsection{Schreibdiphthonge und Doppelvokalschreibungen im Englischen}

Im Englischen gibt es bei morphologisch einfachen Einsilbern folgende Verteilung (Daten nach CELEX, erhoben: alle 4.006 Einsilber des Englischen):

\begin{tabular}{|c|c|c|c|c|c|c|}
\hline \multicolumn{7}{|c|}{ zweiter Bestandteil } \\
\hline \multirow{6}{*}{ 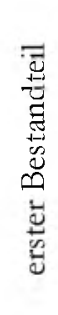 } & & $\mathbf{a}$ & e & $\mathbf{i}$ & $\mathbf{0}$ & $\mathbf{u}$ \\
\hline & $\mathbf{a}$ & 2 & 3 & 84 & 1 & 40 \\
\hline & e & 181 & 126 & 32 & 4 & 9 \\
\hline & $\mathbf{i}$ & 14 & 40 & 0 & 8 & 1 \\
\hline & 0 & 62 & 14 & 33 & 129 & 132 \\
\hline & $\mathbf{u}$ & 5 & 21 & 14 & 2 & 0 \\
\hline
\end{tabular}

Abb. 8: Englische Schreibdiphthonge (nach Berg/Fuhrhop im Erscheinen, S. 450)

Bei der Addition ergeben sich ebenfalls typische erste und typische zweite Bestandteile; bei diesen Zahlen wird deutlich, dass es zunächst um Häufigkeiten geht und nicht um die Ausschließlichkeit bestimmter Schreibdiphthonge wie im Deutschen: 


\begin{tabular}{|l|c|c|c|c|c|}
\hline & a & e & i & o & u \\
\hline erster Bestandteil & 128 & 362 & 63 & 311 & 44 \\
\hline zweiter Bestandteil & 262 & 78 & 163 & 15 & 182 \\
\hline
\end{tabular}

Abb. 9: Erst- und Zweitbestandteile in englischen Schreibdiphthongen (nach Berg/Fuhrhop im Erscheinen, S. 450)

Erste Bestandteile sind $|\mathrm{e}|$ und $|\mathrm{o}|$, zweite Bestandteile $|\mathrm{i}|$ und $|\mathrm{u}|$; in beiden Positionen findet sich $|\mathrm{a}|$. Zu $|\mathrm{i}|$ und $|\mathrm{u}|$ gibt es Varianten als zweite Bestandteile, und zwar $|\mathrm{y}|$ für $|\mathrm{i}|$ und $|\mathrm{w}|$ für $|\mathrm{u}|{ }^{10}$ Beispiele wie say, new usw. werden erst jetzt dazu gezählt und es ergibt sich ein noch deutlicheres Bild:

\begin{tabular}{|l|r|r|r|r|r|}
\hline & a & e & i/y & o & u/w \\
\hline erster Bestandteil & 208 & 403 & 63 & 381 & 44 \\
\hline zweiter Bestandteil & 266 & 78 & 218 & 15 & 312 \\
\hline
\end{tabular}

Abb. 10: Erst- und Zweitbestandteile in englischen Schreibdiphthongen unter Berücksichtigungvon $|y|$ und $|w|$

Im Englischen ist $|\mathrm{o}|$ also ein typischer Erstbestandteil, im Deutschen kommt $\mid$ o $\mid$ so gut wie gar nicht in den Schreibdiphthongen vor (abgesehen von wenigen Ausnahmen, wie moin, ahoi, toi-toi-toi). In Verbindung mit Doppelvokalen ergibt sich, dass in beiden Sprachen nur nicht-zweite Bestandteile verdoppelt werden, im Deutschen $<a$ a, ee, oo $>$, im Englischen $<$ ee, oo $>$. Während das für $<\mathrm{u}>$ und $<\mathrm{i}>$ häufig mit der Form der Buchstaben begründet wird (denn $<\mathrm{uu}>$ und $<$ ii $>$ könnten beim Lesen mit $<w>$ bzw. $<\ddot{u}>$ verwechselt werden), ist dies für $<a>$ wohl nicht mehr zu rechtfertigen, denn im Deutschen kommt <aa> vor, im Englischen hingegen nicht. Die Schreibdiphthongsysteme sind also intern organisiert. Die typischen Zweitbestandteile haben im Englischen Allographen für das Wortende, was Effekte für die graphematische Silbe hat (siehe Fuhrhop/Buchmann/Berg 2011).

Während im Deutschen die Kombinatorik von Erst- und Zweitbestandteilen ausgereizt wird - mit dem Effekt, dass es für einen der Sprechdiphthonge, nämlich / ai / mit <ai> und <ei> zwei Schreibdiphthonge gibt - ist im Englischen die Kombinatorik um das $\langle a\rangle$ herum organisiert. $\langle a\rangle$ kombiniert mit jedem anderen Buchstaben, ob an erster oder zweiter Stelle, hängt systematisch vom jeweils anderen Bestandteil ab, das heißt: Ist $|a|$ Erstbestandteil, kombiniert es nur mit $|\mathrm{u}| /|\mathrm{w}|$ und $|\mathrm{i}| /|\mathrm{y}|$, ist es hingegen Zweitbestandteil, kombiniert es nur mit $|\mathrm{e}|$ und $|\mathrm{o}|$.

$10 \quad$ In Abbildung 4 sind diese Schreibungen bereits als Allographen voneinander erfasst. 


$$
\begin{aligned}
& e-a \\
& o-a \\
& a-u(w) \\
& a-i(y)
\end{aligned}
$$

Abb. 11: System der englischen Schreibdiphthonge

Daneben kommen die Verdopplungen $<$ oo $>$, $<$ ee $>$ und die Kombination $<$ ou> häufig vor.

Bisher konzentrierte sich die Untersuchung auf die schriftlichen Vorkommen im englischen Einsilber; es zeigt sich analog wie im Deutschen eine Beschränkung auf bestimmte Positionen.

Der nächste Schritt ist, die Regelmäßigkeiten zwischen einer Schreibung und einer lautlichen Struktur herauszufinden. In Berg/Fuhrhop (im Erscheinen, S. $452 \mathrm{ff}$.) sind dazu eine Reihe von Beobachtungen und Thesen wiedergeben; hier werden einige wesentliche zitiert. Vorweg sei aber erwähnt, dass es für jeden Vokalbuchstaben grundsätzlich zwei Lese-Varianten gibt, die Venezky (1999, S. 62f.), free und ,checked' nennt, bei Primus (2011) binär und unär, je nachdem, ob die Silbe offen oder geschlossen ist. Hier wird

\begin{tabular}{|c|c|c|c|}
\hline Buchstabe & checked/unär & free/binär & Beispiele \\
\hline a & $\mid \mathfrak{x} /$ & /eI/ & $<$ mat $>-<$ mate $>,<$ mating $>$ \\
\hline $\mathrm{e}$ & $\mid \varepsilon /$ & /i/ & $<$ met $>-<$ mete $>$, $<$ meter $>$ \\
\hline i & $/ \mathrm{I} /$ & /aI/ & $<$ fin $>-<$ fine $>$, $<$ finer $>$ \\
\hline o & $/ a /$ & /ov/ & $<$ hop $>-<$ hope $>$, <hoping $>$ \\
\hline $\mathrm{u}$ & $|\Delta|$ & $/(\mathrm{j}) \mathrm{u} /$ & $<$ cut $>-<$ cute $>,<$ cutie $>$ \\
\hline
\end{tabular}
deutlich, dass finales $\langle\mathrm{e}\rangle$ die Silbe öffnet. Die Korrespondenzen gelten für den trochäischen Fuß, er muss allerdings nicht kanonisch sein, denn die Lesart gilt sowohl für mate als auch für mating.

Abb. 12: Vokalische Graphem-Phonem-Beziehungen im Englischen

Übertragen auf die Diphthonge ist zu fragen, ob von den Einzelbuchstaben auf die Rekodierung im Schreibdiphthong geschlossen werden kann. In Berg/Fuhrhop (im Erscheinen) ist jede Kombination einzeln betrachtet und kommentiert. Bei den im Folgenden genannten ersten Ergebnissen geht es um häufige Korrespondenzen und nicht darum, dass es einzelne andere gibt oder geben könnte. So gibt es ja auch, wie in Abbildung 8 zu sehen, eine Verbindung $<$ ue $>$; dieses steht aber zum Beispiel für einen phonologischen Hiat (fuel), also mit einer internen phonologischen Silbengrenze. Graphematisch wird dies deutlich am untypischen Erstbestandteil $|\mathrm{u}|$, oder es handelt sich um die Vermeidung von $|\mathrm{u}|$ am Wortende wie in blue, true, siehe Abschnitt 4. 
$<a>$ führt als Erstbestandteil im Deutschen zu einer segmentalen Rekodierung $(<\mathrm{ai}>,<\mathrm{au}>)$.

$<\mathrm{a}\rangle$ kann im Englischen an beiden Positionen stehen; in zweiter Position ist aber typischerweise der Einfluss auf die Korrespondenz unspezifisch in dem Sinne, dass es die gleiche ist wie finales $\langle e\rangle$, also die ,freie ${ }^{6}$ Variante.

Beispiel: /ov $/=<_{0} \_\mathrm{e}>$ in hope, note oder $<_{\mathrm{oa}}>$ in coat, soap

Eine solche Beobachtung kann innerhalb der silbischen Schreibungen interpretiert werden. Es würde zumindest in den Graphemauflistungen (Abschnitt 1.1) zu einer Reduktion führen, weil $|a|$ in der zweiten Position bei allen Erstbestandteilen die gleiche Funktion hat, ähnlich wie $<\mathrm{h}>$ im Deutschen mit allen Vokalbuchstaben. Man kann dies als Begründung dafür anführen, dass zum Beispiel Eisenberg (2006, S. 310) keine Grapheme <ah>, <oh> usw. annimmt, wie dies zum Beispiel Nerius (Hg.) (2007, S. 120) tut.

Wenn man nun noch erkennt, dass $|a|$ hier die gleiche Funktion wie finales $\langle\mathrm{e}\rangle$ erfüllt, so kann dies auch weiterführend interpretiert werden, nämlich $|a|$ als graphematischer ,Silbenöffner'. Das ist insofern attraktiv, als es der einzige rundköpfige (aus der Menge $|\mathrm{a}, \mathrm{e}, \mathrm{o}|$, Fuhrhop/Buchmann 2011, S. 82) Buchstabe in der zweiten Diphthongposition im Englischen ist. Wir haben oben schon $\langle\mathrm{e}\rangle$ als, Silbenöffner im Englischen kennengelernt, eben mete wie meat.

$<\mathrm{i}>$ wird sowohl im Deutschen als auch im Englischen als Zweitbestandteil segmental gelesen (voice, aisle, entertain - Kaiser, Feier),

$\left\langle_{0}\right\rangle$ deutet als Erstbestandteil im Englischen auf einen Sprechdiphthong hin (voice, noise, boy, house, foul), sofern es sich nicht um $<00>$ handelt.

Die Bezüge zur Phonologie sind grob die Ergebnisse aus Berg/Fuhrhop (im Erscheinen), zum Teil sind sie hier für die Schreibprinzipien interpretiert. Es scheint also sinnvoll, die englischen Schreibdiphthonge im Zusammenhang mit silbischen und suprasegmentalen Schreibungen zu sehen und sie gerade nicht auf der segmentalen Ebene zu erfassen. Damit zusammen hängt auch die Beobachtung, dass Schreibdiphthonge primär innergraphematisch funktionieren; im Englischen sind die Schreibdiphthonge noch weniger im segmentalen, phonologischen Bezug zu verstehen als im Deutschen; Schreibdiphthonge stehen häufig noch nicht einmal für Sprechdiphthonge. Konkret ist im Sprachvergleich interessant, dass beide Sprachen die gleichen Nur-Zweitbestandteile von Schreibdiphthongen haben, nämlich $|\mathrm{u}|$ und $|\mathrm{i}|$.

\section{Die Doppelkonsonantenschreibung}

Sowohl im Englischen als auch im Deutschen gibt es eine rein morphologische Entstehungsweise von Doppelkonsonanten, nämlich strukturell wenn ein Präfix mit dem gleichen Buchstaben endet wie ein Stamm anfängt (ver- 
reisen, un-natural) oder entsprechend wenn ein Suffix beginnt wie ein Stamm endet (Roh-heit, sudden-ness) bzw. an der Schnittstelle von Komposita (Buchhandlung, wash-house). Diese Fälle kommen im nativen Wortschatz bei Präfixen vor, bei Suffixen sind sie eher selten, auch weil Suffixe häufig mit einem Vokalbuchstaben beginnen. Im lateinischen Wortschatz hingegen sind sie bei Präfixen noch häufiger wie in Immobilie - immobile, illegal, Kollekte - collect usw. Das liegt an der Assimilationsregel, die aus dem Lateinischen übernommen wurde. So kann sich eine Grundform iN- zu im-anpassen, wenn der Stamm mit $<$ m $>$ oder $<p>$ (die phonologisch bilabialen Konsonanten entsprechen) anfängt (immobile, impossible), zu il-vor $<\mathrm{l}>$ (illogical), zu ir-vor $\langle\mathrm{r}\rangle$ (irregular). Sowohl das Deutsche als auch das Englische haben Wörter mit diesen Präfixen reihenweise entlehnt, so auch kol-/col, kom-/com-, kon-/con-, kor/cor-, zum Beispiel collegue, correspond, Kommission, connect, correct usw. Das Auftreten der Konsonantenwiederholung hängt von den jeweiligen Präfixen ab (daher die Annahme von Formen wie $i N$-). Die Doppelkonsonanten sind hier morphologisch bedingt; die Assimilation ist jedoch phonologisch bedingt. In den entlehnten Wörtern kommen überproportional viele Fälle vor. Diese Fälle schließen wir hier zunächst aus, kommen aber später darauf zurück.

\subsection{Die Doppelkonsonantenschreibung im Deutschen}

Im Deutschen gehen wir davon aus, dass Konsonanten doppelt geschrieben werden in den folgenden Kontexten: Zwischen einer betonten Silbe und einer unbetonten Silbe steht phonologisch genau ein Konsonant mit ungespanntem Vokal in der ersten Silbe. ${ }^{11}$ Man kann den gleichen Sachverhalt auch mit Hilfe der Fußstrukturen beschreiben: Eine betonte Silbe in einem Zweisilber (Trochäus) enthält einen binären Kern - dieser kann besetzt sein durch einen ungespannten Vokal und einem Konsonanten, durch einen Diphthong oder durch einen gespannten Vokal (Primus 2011). In der Fußstruktur wird die zweite Kernposition (VC) beim ambisilbischen Konsonanten besetzt; dies entspricht dem Konstrukt des Silbengelenks.

In zweisilbigen Formen ist die Doppelkonsonantenschreibung im Deutschen aufgrund der phonologischen Form gut zu beschreiben; es ergeben sich Schreibungen wie Betten, Männer, boffen, (ver) missen; aufgrund der Morphemkonstanz wird die Doppelkonsonantenschreibung im Einsilber beibehalten und es ergeben sich Schreibungen wie Bett, Mann, hofft, (ver) misst. Für

11 Bei Suffixen ergibt sich eine Besonderheit auch für nicht-hauptbetonte Silben: So werden -in (Freundin) und -nis (Zeugnis) bei nachfolgender weiterer Silbe als -inn(en) und -miss(e) geschrieben; diese verhalten sich auch bezüglich der Morphemkonstanz besonders, denn in der nichterweiterten Form erscheint ein einfacher Konsonantenbuchstabe. Das ist insofern im Zusammenhang mit den graphematischen Füßen zu verstehen: Am Wortende sind die Silben nicht-schwer und werden nicht betont; stehen sie nicht am Wortende, indem noch eine Silbe folgt, können sie eine graphematische Schwere erhalten und entsprechend phonologisch eine Nebenbetonung. 
Zweisilber ergibt sich die Doppelkonsonantenschreibung durch ein silbisches Schreibprinzip, für Einsilber durch ein morphologisches. Die Buchstaben $\langle\mathrm{b}, \mathrm{d}, \mathrm{f}, \mathrm{g}, \mathrm{l}, \mathrm{m}, \mathrm{n}, \mathrm{p}, \mathrm{r}, \mathrm{s}, \mathrm{t}\rangle$ werden verdoppelt, für $\langle\mathrm{k}\rangle$ und $\langle\mathrm{z}\rangle$ gibt es spezielle Gelenkschreibungen, nach Venezky ,Pseudogeminaten', nämlich <ck, tz $>$. Auch <ng $>$ kann als Gelenkschreibung gesehen werden mit einer speziellen phonologischen Regel (für den velaren Nasal). ${ }^{12}$ Die komplexen Grapheme <sch, ch> werden nicht verdoppelt. $<_{w w}>$ kommt lediglich in Strumwelpeter vor. $<\mathrm{v}>$ wird nicht verdoppelt, aber es kommt ebenso wie $\langle\mathrm{h}\rangle$ und $\langle\mathrm{j}\rangle$ nicht an den entsprechenden Stellen vor. $|\mathrm{x}|$ ist im nativen, deutschen Wortschatz überaus selten (Ausnahmen: Hexe, Nixe) und wird allenfalls in Kunstwörtern wie CinemaxX oder Mexx verdoppelt, in Silbengelenkposition (eben Hexe, Nixe) nicht.

\subsection{Die Doppelkonsonantenschreibung im Englischen}

Im Englischen kommt die Doppelkonsonantenschreibung ebenfalls sehr häufig vor. Ganz offenbar ist sie aber im Englischen im Detail anders geregelt als im Deutschen, dazu zwei auffällige Unterschiede: Erstens gibt es mehr Ausnahmen für die entsprechende phonologische Umgebung. So finden sich zwar Schreibungen wie running, killer, wedding usw., in denen die Schreibungen recht analog zum Deutschen ist: Eine betonte erste Silbe mit einem ungespannten Vokal, eine unbetonte zweite Silbe und zwischen den beiden befindet sich phonologisch genau ein Konsonant. Aber: Nicht immer werden hier Doppelkonsonanten geschrieben, denn wir haben Beispiele wie copy, body, city, pity, money, limit usw., in denen nach dieser phonologischen ,Regel' ein Doppelkonsonant geschrieben werden müsste. ${ }^{13}$ Der zweite augenscheinliche Unterschied ist, dass Doppelkonsonanten, die im Zweisilber auftauchen, nicht regelmäßig im Einsilber übernommen werden (running - run/* runn, wohl aber bei <ff, 1l, ss> wie in killing - kill/*kil).

Methodisch sind hier zwei Herangehensweisen denkbar: Erstens werden alle phonologischen Umgebungen herausgesucht, bei denen man - nach Kenntnis des Deutschen - Doppelkonsonantenschreibung vermuten würde. $\mathrm{Zu}$ viele ,Ausnahmen' (copy, city, limit) usw. legen jedoch eine andere Annäherung nahe. Zweitens werden alle vorhandenen Doppelkonsonantenschreibungen untersucht und anschließend wird mit einer neuen These die erste Herangehensweise durchgeführt. Wir suchen also zunächst alle Doppel-

\footnotetext{
$12<\mathrm{ng}>$ taucht in Positionen auf, die typisch für Gelenkschreibungen sind: alleine zwischen zwei Silben nach ungespanntem Vokal wie in fangen, singe, Fänger, Stängel. Es wird in den Einsilber übernommen (fängt, singst oder in den Silbenendrand wie Stängiben). Nur in diesen Positionen wird auch dem Laut [y] phonematischer Charakter zugesprochen. Ansonsten ist [y] als Assimilation zu sehen, wie in danken, denken und möglicherweise auch in ungern, ungerade usw. Für die Pointierung in dieser Sache danken wir Karsten Schmidt.

13 Solche Schreibungen finden sich im Deutschen mit Mini, Limerick usw; sie sind aber auf den Fremdwortbereich zu beschränken.
} 
konsonantenschreibungen heraus und führen unsere Untersuchung durch. Die lateinischen haben wir ebenso wie morphologische Grenzen des Typs sudden-ness zunächst heraussortiert.

\subsubsection{Doppelkonsonanten im Englischen: suprasegmental und morphologisch}

Primus (2011, S. 7) geht davon aus, dass die Ambisyllabizität in kanonischen Wortformen systematischer ist als in nicht-kanonischen: Kanonische Füße sind wie gesagt trochäische Füße mit einer Schwa- bzw. $<\mathrm{e}>$-Silbe. Betrachtet man die nicht-kanonischen mit, so drängt sich folgende Beobachtung auf: Die graphematischen Doppelkonsonanten zeigen morphologische Komplexität, was wir an der Gegenüberstellung von copy und floppy, sunny erklären: In copy hat $-y$ keine morphologische Funktion, in floppy, sunny handelt es sich um morphologische Bildungen flop - floppy, sun - sumny. Im Folgenden sind die Doppelkonsonanten in einem ersten Schritt morphologisch geordnet nach Suffixen. Die Suffixe beginnen mit einem Vokalbuchstaben, sodass mit dem morphologischen Prozess eine graphematische Silbengelenkposition entsteht (zu -ed und -le siehe unten).

\begin{tabular}{|c|c|c|c|c|c|c|c|c|}
\hline \multirow[b]{2}{*}{ DK } & \multicolumn{3}{|c|}{ kanonische Füße } & \multicolumn{4}{|c|}{ nicht-kanonische Füße } & \multirow[b]{2}{*}{ weitere } \\
\hline & vor $-e r$ & vor $-e d$ & vor $-l e,-e l$ & vor -ing & vor $-y$ & vor -ish & vor -able & \\
\hline $\mathrm{bb}$ & rubber & jobbed & $\begin{array}{l}\text { bubble, } \\
\text { pebble }\end{array}$ & $\begin{array}{l}\text { jobbing, } \\
\text { stabbing, } \\
\text { throbbing }\end{array}$ & bobby & $\begin{array}{l}\text { rubbish, } \\
\text { snobbish }\end{array}$ & clubbable & rabbit \\
\hline dd & bidder & nodded & paddle & bidding & daddy & faddish & biddable & $\begin{array}{l}\text { redden, } \\
\text { madden, }\end{array}$ \\
\hline$g g$ & $\begin{array}{l}\text { jogger, } \\
\text { stagger }\end{array}$ & rugged & smuggle & leggings & $\begin{array}{l}\text { twiggy, } \\
\text { buggy }\end{array}$ & piggish & huggable & $\begin{array}{l}\text { thuggery, } \\
\text { druggist }\end{array}$ \\
\hline $\mathrm{mm}$ & slimmer & slammed & trammel & swimming & $\begin{array}{l}\text { mommy, } \\
\text { slummy }\end{array}$ & slimmish & swimmable & summary \\
\hline nn & $\begin{array}{l}\text { planner, } \\
\text { beginner }\end{array}$ & planned & tunnel & running & sunny & Finnish & $\begin{array}{l}\text { winnable, } \\
\text { runnable }\end{array}$ & nunnery \\
\hline PP & hopper & wrapped & grapple & shipping & $\begin{array}{l}\text { happy, } \\
\text { floppy }\end{array}$ & snappish & unflappable & happen \\
\hline $\mathrm{tt}$ & fatter & committed & bottle & fitting & $\begin{array}{l}\text { Scotty, } \\
\text { pretty }\end{array}$ & $\begin{array}{l}\text { skittish, } \\
\text { sluttish }\end{array}$ & $\begin{array}{l}\text { forgettable, } \\
\text { regrettable }\end{array}$ & $\begin{array}{l}\text { forgotten, } \\
\text { written }\end{array}$ \\
\hline
\end{tabular}

Abb. 13: Doppelkonsonanten - sortiert nach Suffixen

Der nächste logische Schritt ist dann, zu prüfen, ob sich sämtliche Suffixe des Englischen, die mit einem vokalischen Buchstaben beginnen, entsprechend verhalten. Von den häufigen Suffixen, die im Englischen vorkommen, 
fehlen hier -ic, -ous und -ism. Zumindest -ic und -ous zeichnen sich dadurch aus, dass sie den Akzent verschieben (zu den Akzentverhältnissen bei Affixen vgl. Plag 2002, S. 146, siehe auch S. 120-122). Sie ergeben dann phonologisch keinen kanonischen Fuß mehr, sie sind noch nicht einmal mehr Trochäen und das drückt sich auch graphematisch aus. Bezüglich der Doppelkonsonanz verhält sich auch -ee in refere $e^{14}$ markiert, es zieht den Akzent auf sich und verhält sich damit einsichtigerweise anders als referring, siehe unten. Die graphematische Doppelkonsonanz wird nur gezeigt, wenn die phonologischen Fußstrukturen das auch hergeben.

Nun sind aber nicht alle Doppelkonsonantschreibungen so zu beschreiben, viele Ausnahmen finden sich bei -er und -le/el, eben den kanonischen Füßen (summer, hammer, little, tunnel) - hier ist die Schreibung also suprasegmental begründet mit den beiden Ausnahmen proper, leper.

In den nicht-kanonischen Füßen findet sich hingegen die morphologische Fundierung wie an Beispielen wie limit, copy, tenable usw. zu sehen ist. Nehmen wir als Beispiele to finish - Finnish, tenable - munable: Wenn -ish ein Suffix ist, dann wird der Konsonant verdoppelt, sofern er zwischen betonter und unbetonter Silbe vorkommt und der vorausgehende Vokal ungespannt ist. In finish (Verb) und blemish liegt - zumindest synchron betrachtet - kein Suffix vor, sodass hier -ish keine morphologische Funktion hat. Finnish (Herkunftsbezeichnung) hingegen ist zweimorphemig, mit Bezug auf Finland (englische Schreibweise) wird der Doppelkonsonant begründet. Gleiches gilt für Wörter wie tenable, das regulär mit einem $<_{\mathbf{n}}>$ geschrieben wird, da eine Ableitungsbasis wie *(to) ten fehlt, aber runnable, winnable mit den Basen to mu, to min.

Unter den Wörtern, die auf -er enden, sind ebenfalls einige, die phonologisch keine Trochäen sind, sondern auf der zweiten Silbe betont sind, und zwar Wörter wie confer, prefer, refer. Graphematisch wird dies deutlich bei weiteren Silben wie conferring, preferring, referring (im Gegensatz zu offering usw.) - hier ist auch graphematisch eine andere Fußstruktur zu erkennen. Die Doppelkonsonanz zeigt geradezu die betonte Silbe an, in referring ist die zweite Silbe die betonte (oder schwere), in offering ist es die erste (Carney 1994, S. 123).

In Abbildung 13 sind -le und - $e$ in einer Spalte aufgeführt: Dies hat den Hintergrund, dass nach $<\mathrm{m}, \mathrm{n}$, r $>$ ausschließlich -el auftaucht, nach den anderen Konsonanten aber -le. Allerdings ist hier noch einmal Anlass, über den Begriff des Silbengelenks zu reflektieren: Bei -le ,beginnt ${ }^{c}$ das Suffix graphematisch mit einem Konsonantenbuchstaben, lautlich liegt ein silbischer Konsonant vor, ,beginnt ${ }^{c}$ also mit dem Silbenkern, weil der silbische Konsonant der Silbenkern ist. ${ }^{15}$ Mit -ed ist häufig nur eine graphematische Silbe

\footnotetext{
14 So wird bei committee ein doppelter Konsonant geschrieben, hier ist aber nicht das Suffix betont, sondern die Silbe davor.

15 Die Schreibung -el beginnt sowohl graphematisch als auch phonologisch mit dem Silbenkern. Venezky (2004, S. 143) bezeichnet die Schreibung -le als, reversed spellings.
} 
gemeint, sie enthält einen Silbenkern, das Suffix beginnt mit einem vokalischen Buchstaben. Es ist aber lautlich nicht immer silbisch (vgl. Locked, stepped). Die Doppelkonsonantenschreibung verhält sich in beiden Fällen gleich, unabhängig davon, ob es sich nur um eine graphematische Silbe oder auch um eine phonologische handelt und unabhängig davon, ob sie nur graphematisch oder nur phonologisch mit dem Silbenkern beginnt. Für eine Silbengelenkschreibung scheint es also geradezu gleichgültig, ob es sich um eine phonologische Gelenkstelle (little) oder um eine graphematische (stepped) handelt.

\subsubsection{Doppelkonsonanten im Englischen und Morphemkonstanz}

Bis hierher wurden die Doppelkonsonanten betrachtet, die ausschließlich intersyllabisch auftreten. Oben ist schon angedeutet, dass einige der Doppelkonsonanten doch so etwas wie Morphemkonstanz aufweisen, nämlich $l /$, $f f$, ck, ss: to kill, staff, to pack, kiss. Wir möchten sie hier einzeln erläutern:

Für <ss> liegt eine Erklärung auf der Hand: das Flexionssystem. So ist nämlich zu beobachten, dass es im Englischen keine morphologisch einfachen Wörter gibt, die mit $|\mathrm{s}|$ enden - die Begründung dafür könnte sein, dass $s$ am Wortende nur auftritt, wenn es morphologisch (als Plural ${ }^{16}$ oder 3. Ps. Sg. Präs.) interpretiert werden soll. Als Ausweichschreibungen haben sich $<$ ss $>$ nach einfachem Vokalgraphem (kiss, mass) bzw. finales |e| nach komplexem Vokalgraphem (house, noise) etabliert. ${ }^{17}$

$<11>$ verhält sich noch anders: Es wird nicht immer am Silbenrand beibehalten wie in killer - to kill, sondern offenbar nur, wenn es auch die betonte/schwere Silbe ist wie in $f u l(l)$ also fuller - full, dagegen beautiful - beautifully. Der Fall erinnert an das deutsche Lebrerin - Lebrerinnen und zum Teil auch an den oben genannten Unterschied zwischen offering und referring; gemeinsam ist allen Fällen, dass die Doppelkonsonantenschreibungen auch etwas über die vorangehende Silbe aussagen; sie kennzeichnen sie als schwer.

Dass $<\mathrm{ll}>$ und $<\mathrm{ff}>$ im Einsilber vorkommen, ist nicht direkt zu erklären. An dieser Stelle ist allerdings eine Parallele in der Geschichte des Deutschen zu sehen: bei $\langle\mathrm{ff}\rangle$ und $<\mathrm{ll}>(\mathrm{und}\langle\mathrm{tt}\rangle)^{18}$ hat sich die ausschließliche Interpretation als Silbengelenkschreibung als letztes durchgesetzt (Tieffe, schuff, alls, Gewallt usw., Voeste 2009, S. 10). Nach Voeste ist das Entscheidende die Schmalheit der Buchstaben, $<\mathrm{mm}>$ als besonders breite Buchstaben findet sich entsprechend ausschließlich als Gelenkschreibung.

\footnotetext{
16 Der Genitiv wird im Englischen typischerweise mit einem Apostroph geschrieben.

17 Die einzige Ausnahme hierzu ist lens, eine Schreibung, die unter Fotofachleuten im Internet diskutiert wird.

$18<\mathrm{tt}\rangle$ ist im Englischen auch zu finden ohne die übliche Systematik wie in $\langle$ butt $>,<$ nett $>$, siehe Abschnitt 3.2.3.
} 
Die Pseudogeminanten (Venezky 2004, S. 144) <ck $>$ und $<$ tch $>$ (match - matches vs. church - churches) ${ }^{19}$ werden im Einsilber auch beibehalten. Die Pseudogeminate $<\mathrm{dg}>$ hingegen tritt nur in graphematischen Zweisilbern auf (bridges - bridge); sie verlangt nach einem $<\mathrm{e}>$ am Ende. Dabei steht $<\mathrm{dg}>$ in graphematischer Konkurrenz $\mathrm{zu}<\mathrm{gg}>$, nämlich als Geminatenschreibung von $<\mathrm{g}>$. Die Regularität ist die folgende: Wenn $<\mathrm{g}>$ dem velaren Plosiv /g/ entspricht, wird das Graphem regulär zu $<\mathrm{gg}>$ verdoppelt (begging). Wenn $<\mathrm{g}>$ jedoch der Affrikate / $\mathrm{d}_{3} /$ entspricht, kommt die Gelenkschreibung $<\mathrm{dg}>$ zum Zuge (hedging), allerdings nur unter den folgenden Bedingungen: Im Silbengelenk wird $<\mathrm{dg}>$ geschrieben wie in budget, edges usw. Im Endrand wird es beibehalten, graphematisch bleibt es aber wie gesagt ein Silbengelenk, da immer $<\mathrm{e}>$ folgt. Im Anfangsrand steht einfaches $\langle\mathrm{g}\rangle$ (gender), allerdings nur vor $/ \mathrm{i} / \mathrm{und} / \varepsilon /$. Vor $/ \mathrm{a}, \mathrm{o}, \mathrm{u} / \mathrm{steh}$ hingegen $<\mathrm{j}>($ jam, jolly, jungle $)$.

\begin{tabular}{|l|l|c|}
\hline & primär & SG \\
\hline Deutsch: $/ \mathrm{ts} /$ & $<\mathrm{z}>$ & $<\mathrm{tz}>$ \\
\hline Englisch: $/ \mathrm{d}_{3} /$ & $<\mathrm{g}>,<\mathrm{j}>$ & $<\mathrm{dg}>$ \\
\hline English: $/ \mathrm{t} \mathrm{g} /$ & $<\mathrm{ch}>$ & $<\mathrm{tch}>$ \\
\hline
\end{tabular}

Abb. 14: Besondere Gelenkschreibungen (Pseudogeminaten)

Die Silbengelenkschreibung ist in vielen Fällen die Verdopplung des Konsonantenbuchstabens, in anderen Fällen gibt es besondere Schreibung $(d g$, $t c h, c k, n g)$. Sowohl die besonderen Schreibungen als auch $l l, f f, s s$ werden im Einsilber beibehalten. Alle anderen Einsilber werden mit einfachem Konsonanten geschrieben (run, big, swim, fat). Wenn man nun das System versucht zu vervollständigen, so ist auffällig, dass $<_{\mathrm{v}}>$ selten verdoppelt wird, obwohl es in der entsprechenden (phonologischen) Position vorkommt wie in loving, living; es sind nicht übermäßig viele Fälle. Die Verdopplung von $|\mathrm{v}|$ gibt es zwar, aber sie ist eher selten und eine neuere Erscheinung.

Seit dem 19. Jahrhundert kommen Wörter mit $\langle\mathrm{vv}\rangle$ häufiger vor (so auch Crystal 2005, S. 263), und zwar regelkonform nach Kurzvokal zwischen betonter und unbetonter Silbe. Das OED listet folgende Beispiele, die auch im BNC- sowie COCA-Korpus vorkommen:

beviy, bivry, bovver, bruvver, chavey, chivey, civey, divvers, divy, flivver, laviy, luvvie, mivy, muvver, navy, nevry, revring, savy, sivvens, skivry, spivrish.

Es gibt die Schreibung also, aber nicht systematisch.

19 Auch in rich, attach wäre eine Schreibung mit <tch $>$ angemessen, es sind die einzigen Ausnahmen in CELEX. 


\begin{tabular}{|l|c|c|c|c|c|c|c|}
\hline & $\begin{array}{c}\text { savvy } \\
(1785)\end{array}$ & $\begin{array}{c}\text { civvy } \\
(1889)\end{array}$ & $\begin{array}{c}\text { bevvy } \\
(1889)\end{array}$ & $\begin{array}{c}\text { bruvver } \\
(1898)\end{array}$ & $\begin{array}{c}\text { chivvy } \\
(1918)\end{array}$ & $\begin{array}{c}\text { lavvy } \\
(1961)\end{array}$ & $\begin{array}{c}\text { luvvie } \\
(1988)\end{array}$ \\
\hline BNC & 33 & 27 & 7 & 8 & 11 & 15 & 7 \\
\hline COCA & 2.567 & 1 & 1 & 0 & 5 & 0 & 0 \\
\hline
\end{tabular}

Abb. 15: Vorkommen von $\langle\mathrm{vv}\rangle$

Interessant sind aber die Paare love - loving, give - giving usw: Die Schreibung ist in sich stringent; beide Zweisilber bilden Füße, in denen die Schreibung suggeriert, dass der Vokal binär ist, obwohl dies nicht der prosodischen Struktur entspricht. In Abschnitt 4 wird deutlich, dass $\langle\mathrm{v}\rangle$ im Englischen kein gutes Wortende ist (so eben genitive im Englischen und Genitiv im Deutschen); das $\langle\mathrm{e}\rangle$ in give, live usw. wäre also damit zu begründen. Dass dies aber auch für Formen wie loving, lover (statt *loving, *livving usw.) durchgehalten wird, ist erstaunlich. Ein einzelnes Beispiel mit einem anderen Konsonanten, das sich ebenso verhält, findet sich mit come - coming (nicht * comming); graphematisch erscheint die Paarung konsequent - offene Silbe bei co-me, einfacher Konsonant bei coming.

\subsubsection{Doppelkonsonanten in englischen Einsilbern} ohne Morphemkonstanz

Oben sind die Besonderheiten von $<1 \mathrm{l}$, ff, ss $>$ beschrieben worden. Nach allem, was bisher festgestellt wurde, dürfte es die anderen Konsonanten nicht in doppelter Schreibung im Endrand von Einsilbern geben. Einige finden sich aber dennoch:

bb: ebb

dd: add, odd

gg: egg

nn: banns, inn

rr: whirr, err, burr, parr, purr

tt: butt, mitt, nett, putt, sett, watt

$z z:$ buzz, fizz, frizz, fuzz, jazz

Abb. 16: Doppelkonsonanten in englischen Einsilbern ohne Morphemkonstanz

Dies sind die einzigen Doppelkonsonanten, die CELEX für den Einsilber listet. Nach Jespersen gibt es eine orthographische Regel, nach der ein (Inhalts-) Wort eine Mindestlänge von drei Buchstaben aufweisen sollte (1928 [1909], \$4.96). Illustrieren kann man das an dem Minimalpaar egg vs. leg: Letzteres weist bereits drei Buchstaben auf, eine Verdopplung des $\langle\mathrm{g}\rangle$ ist hier nicht nötig. Anders verhält es sich bei egg. Endet ein Wort, das nur zwei Buchstaben aufweist, auf einen Konsonanten, so wird dieser verdoppelt. En- 
det ein Wort hingegen auf einen Vokal, so wird finales $\mid$ e $\mid$ angefügt: foe, roe, die, lie, cue, due. Die Frage bei einem solchen Prinzip ist natürlich, wie systematisch es wirkt und ob dies die einzige Begründung ist. Offenbar gibt es im Englischen so etwas wie , verbotene' Wortenden (siehe Abschnitt 4), zum Beispiel $|\mathrm{u}|$, das auch bei mehrbuchstabigen Wörtern ein $|\mathrm{e}|$ verlangt wie in true und blue.

Die Fälle mit <rr $>$ sind selten, außerdem gibt es Varianten mit einem $<\mathbf{r}>$ (bur, whir . Es sind Einzelfallanhäufungen, so könnte für err eben auch eine Drei-Buchstaben-Regel gelten. Für burr ist im Wörterbuch Onomatopoesie angegeben; es wird übersetzt mit „breïge Aussprache (von R) “ (Langenscheidt/Collins 2008), eine Verallgemeinerung, wie Carney (1994, S. 187) sie annimmt, ist hier nicht direkt zu sehen. Auch bei $<z z>$ liegt der Gedanke nahe, dass es sich bei bu $\approx$ und fi\% um Onomatopoetika handelt: Sie bezeichnen Laute (brummen, zischen). Frizz, furq sowie jazz sind hingegen späte Entlehnungen aus dem 17. bzw. 19. Jahrhundert und somit neuere Schreibungen. Es sind wenige Fälle.

Die Fälle mit $<\mathrm{tt}>$ beschreibt Jespersen (1928 [1909], \$4.97 und Venezky 1999, S. 13) als Homonymiedifferenzierungen, wobei jeweils dem Substantiv durch ein Mehr an graphischer Substanz mehr Gewicht zukommt: but vs. butt, net vs. nett, put vs. putt, set vs. sett. Dieses Prinzip muss allerdings noch in der Systematik bewiesen werden; an dieser Stelle wirkt die Begründung von Jespersen ad hoc. Allerdings spricht im Englischen viel für ein Heterographieprinzip wie zum Beispiel die systematische doppelte Kodierungsmöglichkeit von Langvokalen und Diphthongen (/i/ als <ea $>,<e e>$ sowie die oben genannte Regelmäßigkeit, dass $|a|$ als zweiter Diphthongbestandteil die gleichen Auswirkungen hat wie $|\mathrm{e}|$ in der zweiten graphematischen Silbe usw.).

\subsection{Doppelkonsonanten - Zusammenfassung}

Bei der Doppelkonsonantenschreibung im Englischen ist zunächst zu unterscheiden, ob es sich um kanonische oder nicht-kanonische Füße handelt. Bei den kanonischen (trochäisch mit $<\mathrm{e}>$ - oder Schwa-Silbe) sind die Doppelkonsonanten suprasegmental bedingt, hier ähnelt das Englische dem Deutschen. Bei den nicht-kanonischen Füßen, hier insbesondere trochäische Füße mit betonbaren, potenziell schweren zweiten Silben, zeigen die Doppelkonsonanten primär morphologische Struktur: Wenn Doppelkonsonanten geschrieben werden, dann ist das Wort morphologisch komplex. Hieraus ergibt sich, dass suprasegmental bedingte Doppelkonsonanten nicht im Einsilber erscheinen, die eigentliche Bedingung fällt weg.

Die große Menge von lateinischen Wörtern des Typs immobile, illegal, colloquial usw., die das Englische aufgenommen hat, fügt sich somit zum Teil gut ein, denn auch sie sind morphologisch komplex. Sie stehen allerdings 
für eine andere prosodische Struktur und stehen im Widerstreit mit den Betonungsverhältnissen, die zum Beispiel beautiful - full aufweisen. Allerdings ist die zweite Menge die sehr viel kleinere. Möglicherweise gewinnt hier die morphologische Strukturierung die Oberhand. Das ist daher so interessant, weil auf den ersten Blick das Englische gerade bei den Doppelkonsonanten die Morphemkonstanz nicht wie im Deutschen aufweist - keine Morphemkonstanz, aber das Anzeigen von morphologischer Struktur; ein weiteres morphologisches Schreibprinzip.

Nun ist es überraschend, dass sich unterschiedliche Regeln für kanonische und nicht-kanonische Füße zeigen, obwohl beide hier untersuchten im Wesentlichen trochäische Füße sind. Betrachten wir daher noch einmal die Endungen der kanonischen Füße: -er, -en, -el/-le, -ed. Drei der vier Endungen sind silbische Sonoranten; wie im Deutschen sind sie im Prinzip mögliche Suffixe, aber durchaus auch in mehrerer Hinsicht. So kann er sowohl Nomen-agentis-Suffix als auch Komparativsuffix sein, -en Partizip-II-Endung und Verbwortbildungssuffix (red - to redden, sad - to sadden) sein. Insbesondere -er und -en sind multifunktional in der Morphologie des Englischen. -ed hingegen hat in den vorgefundenen Formen immer die gleiche Funktion - Suffix für Verben (Imperfekt und Partizip II). So sind die Doppelkonsonanten vor -ed quantitativ auch morphologisch zu deuten. Das ist auch deswegen interessant, weil -ed ja nicht immer auch phonologisch einer Silbe entspricht. Die Schreibung ist hier konsequent - sowohl kanonische Füße als auch morphologische Struktur. Bei relativ ,eindeutigen' Suffixen wird also auch bei den kanonischen Füßen morphologische Struktur gezeigt. Die anderen Endungen (die silbischen Sonoranten) sind auch im Deutschen nicht zu unrecht häufig als Pseudosuffixe (Eisenberg 2006, S. 164) bezeichnet worden.

\section{Verbotene Wortenden}

Im Englischen scheinen einige Buchstaben nicht am Wortende vorzukommen. Sicherlich haben verschiedene Nichtvorkommen verschiedene Gründe, wie wir auch gleich sehen werden. Aber das Schreibsystem des Englischen ist so komplex, dass wir den Gedanken hier kurz verfolgen wollen, denn im Deutschen scheint ein solches Prinzip nicht explizit zu wirken. Wenn man im Deutschen systematisch nach Buchstaben am Ende sucht, kommt als Ergebnis heraus, dass $|\mathrm{v}|$ und $|\mathrm{w}|$ praktisch nicht vorkommen, $|y|$ und $|c|$ betrachten wir ohnehin als Fremdgrapheme. Alle anderen Buchstaben kommen vor. Dass $|\mathrm{v}|$ und $|\mathrm{w}|$ nicht vorkommen, ist auch schon aus anderer Richtung aufgefallen in Fällen wie doof, elf, wwölf vs. brav (siehe Eisenberg 2006, S. 428). Wenn man das Gleiche für das Englische abfragt, dann ergeben sich eine Reihe von Enden, die nicht vorkommen: $v, u, s, i, c$. 
Bei $v, u, s, c$ kann ein $|\mathrm{e}|$ angehängt werden (hive, true, voice, house), das $\langle\mathrm{e}\rangle$ hat dabei bis auf bei $\langle\mathrm{c}\rangle$ keinen Einfluss auf die Lautung. Statt $u$ kann wenn noch ein anderer Vokalbuchstabe vorhanden ist, also im Schreibdiphthong - ein $|\mathrm{w}|$ geschrieben werden (show, now, how, new), für $i$ kann auch unabhängig von einem anderen Vokalbuchstaben ein $|\mathrm{y}|$ geschrieben werden (shy, why, happyy, lady). $<_{\mathrm{s}}>\mathrm{kann}$ in bestimmten Umgebungen (alleine nach ungespanntem Vokal) auch verdoppelt werden (kiss, miss, mass, boss), lediglich für $\langle\mathrm{v}\rangle$ gibt es nur eine Variante, um es als Wortende zu vermeiden, nämlich $\langle\mathrm{e}\rangle$, wenn man davon ausgeht, dass bei $\langle\mathrm{c}\rangle$ die Lautung als $/ \mathrm{s} /$ statt $/ \mathrm{k} /$ gesichert wird (service, voice vs. das Suffix $-i c$ wie in electric). Die Verhinderung von bestimmten Wortenden ist aber auffällig.

\section{Morphologische Prinzipien: Komplexitätsanzeige auch im Deutschen?}

Es hat sich gezeigt, dass die Doppelkonsonantenschreibung im Englischen zum Teil morphologisch organisiert ist; insbesondere in den nicht-kanonischen Fußstrukturen wird das deutlich. Im Deutschen sind wir davon ausgegangen, dass sie im Zweisilber phonographisch (silbisch, suprasegmental) herzuleiten ist. Allerdings haben die meisten dieser Zweisilber eben genau die Struktur: ein Stamm mit einem vokalisch anlautenden Derivationssuffix, also Schnimmer, Rettung, eckig, spöttisch oder eben mit einem Flexionssuffix Männer, Gottes, schneller, und zwar im nativen Wortschatz unabhängig von der Form des Suffixes, zum Fremdwortbereich siehe Eisenberg (2011, S. $335 \mathrm{ff}$.).

Insofern könnten auch die Doppelkonsonanten im Deutschen so interpretiert werden - das Zeigen der morphologischen Struktur wirkt hier implizit, mit der sehr ähnlichen Ausnahme bei -er wie in Hammer, Sommer, Kammer usw., die nicht direkt morphologisch interpretiert werden können, aber schon häufiger aus verschiedenen Gründen als Pseudosuffixe beschrieben worden sind. Auslautendes - $e$ kann ähnlich interpretiert werden, wie in Platte, Mappe usw., ebenso -el wie in Triffel, Waffel usw. In der vorliegenden Ausdrucksweise entspricht dem Folgendes: Die Doppelkonsonantenschreibung ist im Deutschen explizit nach dem suprasegmentalen Prinzip geregelt, implizit aber auch morphologisch.

\section{Zusammenfassung}

Zunächst wurden einzelne Schreibprinzipien und ihr Zusammenspiel erläutert. Daraufhin wurden die Schreibdiphthonge und die Doppelkonsonanten untersucht, die erstens in beiden Sprachen deutlich unterschied- 
lich organisiert sind und die zweitens ein interessantes Zusammenspiel der Prinzipien zeigen. Sowohl die deutschen als auch die englischen Schreibdiphthonge sind zunächst innergraphematisch organisiert; es gibt typische erste und typische zweite Bestandteile. Während im Deutschen die beiden Erstbestandteile mit den beiden Zweitbestandteilen kombinieren, ergibt sich im Englischen ein System, das sich um das $|\mathrm{a}|$ herumgruppiert. Aus dieser Beobachtung lassen sich Beziehungen zur Lautstruktur aufstellen, aber erst mit der besonderen Betrachtung des Gesamtsystems und seiner innergraphematischen Strukturierung.

Eine erste Annäherung über eine rein phonographische Fundierung der Doppelkonsonantenschreibung im Englischen ist gescheitert; sie funktioniert nur für kanonische Füße. Für nicht-kanonische Füße muss neben der notwendigen phonographischen Bedingung (erste Silbe ungespannter Vokal und zwischen dem ersten und zweiten Silbenkern genau ein Konsonant) eine morphologische erfüllt sein: In der zweiten Silbe steckt ein vokalisch anlautendes, unbetontes Suffix. Umgekehrt formuliert zeigt der Doppelkonsonant die morphologische Struktur. Dies wiederum kann auch auf das Deutsche übertragen werden. In den meisten Fällen zeigt die Doppelkonsonantenschreibung auch im Deutschen eine morphologische Struktur, denn der typische Zweisilber, in dem sich Doppelkonsonanten zeigen, ist morphologisch komplex. Das Deutsche geht hier noch einen Schritt weiter und zeigt die (flexions-)morphologische Potenz auch im Einsilber.

Es hat sich in allen betrachteten Fällen gezeigt, dass es innergraphematische Schreibprinzipien im Deutschen und Englischen gibt und dass die morphologischen Schreibungen weit über das Prinzip der Morphemkonstanz hinausgehen.

\section{Literatur}

Becker, Thomas (1996): Zur Repräsentation der Vokallänge in der deutschen Standardsprache. In: Zeitschrift für Sprachwissenschaft 15, S. 3-21.

Berg, Kristian/Fuhrhop, Nanna (i.Ersch.): Silbenkernschreibungen im Deutschen und Englischen. Ersch. in: Linguistische Berichte 228, S. 443-466.

BNC $=$ Davies, Mark (2008ff.): British National Corpus. 100 million words, UK, 1980s1993. Internet: http://corpus.byu.edu/bnc/ (Stand: 16.09.2011).

Carney, Edward (1994): A survey of English spelling. London/New York.

CELEX = Bayen, R. Harald/Piepenbrock, Richard/Gulikers, Leon (1995): The CELEX lexical database (CD-ROM). Philadelphia.

COCA $=$ Davies, Mark (2008 ff.): The Corpus of Contemporary American English. 410+ million words, 1990-2011. Internet: www.americancorpus.org/ (Stand: 16.09.2011).

Crystal, David (2005): The Cambridge encyclopedia of the English language. 2., nachgedr. Aufl. Cambridge u.a. 
Dewey, Godfrey (1970): Relative frequency of English spellings. New York.

Eisenberg, Peter (2006): Grundriss der deutschen Grammatik: Das Wort. 3., durchges. Aufl. Stuttgart/Weimar.

Eisenberg, Peter (2011): Das Fremdwort im Deutschen. Berlin/New York.

Evertz, Martin (2011): Foot structure in non-linear graphematics - experimental evidence from German. Ms. Köln.

Fuhrhop, Nanna/Buchmann, Franziska (2009): Die Längenhierarchie. Zum Bau der graphematischen Silbe. In: Linguistische Berichte 218, S. 127-155.

Fuhrhop, Nanna/Buchmann, Franziska (2011): Buchstabenformen und ihre Relevanz für eine Schriftgrammatik. Erwiderung auf einige Thesen von Oliver Rezec. In: Linguistische Berichte 225, S. 77-88.

Fuhrhop, Nanna/Buchmann, Franziska/Berg, Kristian (2011): The letter length hierarchy: evidence from German and English. In: Written Language and Literacy 14.2 , S. 275-292.

Giegerich, Heinz J. (1992): English phonology: an introduction. Cambridge.

Hanna, Paul R. et al. (1966): Phoneme-grapheme correspondences as cues to spelling improvement. Washington, DC.

Jespersen, Otto (1928 [1909]): A modern English grammar I: sounds and spelling. 4. Aufl. Heidelberg. [1. Aufl. Heidelberg 1909].

Kessler, Brett/Treiman, Rebecca (2001): Relationships between sounds and letters in English monosyllables. In: Journal of Memory and Language 44, S. 592-617.

Ladefoged, Peter (2006): A course in phonetics. 5. Aufl. Boston.

Langenscheidt/Collins (2008): Langenscheidt Collins Großwörterbuch Englisch. 6., neubearb. Aufl. Berlin u.a.

Meisenburg, Trudel (1996): Romanische Schriftsysteme im Vergleich. Eine diachrone Studie. (= ScriptOralia 82). Tübingen.

Müller-Lancé, Johannes (2006): Latein für Romanisten. Ein Lehr- und Arbeitsbuch. Tübingen.

Neef, Martin (2005): Die Graphematik des Deutschen. (= Linguistische Arbeiten 500). Tübingen.

Nerius, Dieter (Hg.) (2007): Deutsche Orthographie. 4., neu bearb. Aufl. Hildesheim.

OED $=$ The Oxford English Dictionary. The definite record of the English language: The definitive record of the English language. Internet: www.oed.com/ (Stand: 09.09.2011).

Plag, Ingo (2002): Word-formation in English (Entwurf). Internet: www.scribd.com/doc/ 4045836/WordFormation-in-English (Stand: 16.09.2011).

Primus, Beatrice (2010): Strukturelle Grundlagen des deutschen Schriftsystems. In: Bredel, Ursula/Müller, Astrid/Hinney, Gabriele (Hg.): Schriftsystem und Schrifterwerb: linguistisch - didaktisch - empirisch. (= Germanistische Linguistik 289). Tübingen, S. $9-45$. 
Primus, Beatrice (2011): The graphematic foot in English and German. Ms. Köln.

Restle, David/Vennemann, Theo (2001): Silbenstruktur. In: Haspelmath, Martin et al. $(\mathrm{Hg}$.): Language typology and language universals. Teilbd. 2. (= Handbücher zur Sprach- und Kommunikationswissenschaft 20.2). Berlin u.a., S. 1310-1336.

Roemheld, Friedrich (1955): Die Längenbezeichnungen in der deutschen Rechtschreibung. In: Der Deutschunterricht. Beiträge zu seiner Praxis und wissenschaftlichen Grundlegung 7, 3, S. 71-82.

Venezky, Richard L. (1999): The American way of spelling. The structure and origins of American English orthography. New York.

Venezky, Richard L. (2004): In search of the perfect orthography. In: Written Language and Literacy 7.2, S. 139-163.

Voeste, Anja (2009): Zwischen Wort und Zeichen. Die graphische Markierung suprasegmentaler Einheiten in der Frühen Neuzeit. In: Zeitschrift für Germanistische Linguistik 37, 1, S. 1-14. 